\title{
Theory and calibration of JWL and JWLB thermodynamic equations of state
}

\author{
E. L. Baker ${ }^{1}$, D. Murphy ${ }^{1}$, L. I. Stiel ${ }^{2} \&$ E. Wrobel ${ }^{1}$ \\ ${ }^{1}$ US Army Armament Research Development and Engineering Center, \\ USA \\ ${ }^{2}$ New York Polytechnic University, USA
}

\begin{abstract}
Structure geometric configuration and response can be strongly coupled to blast loading particularly for close-in blast loading configurations. As a result, high rate continuum modeling is being increasingly applied to directly resolve both the blast profiles and structural response. In this modeling, the equation of state for the detonation products is the primary modeling description of the work output from the explosive that causes the subsequent air blast. The JonesWilkins-Lee (JWL) equation of state for detonation products is probably the currently most used equation of state for detonation and blast modeling. The Jones-Wilkins-Lee-Baker (JWLB) equation of state is an extension of the JWL equation of state that we commonly use. This paper provides a thermodynamic and mathematical background of the JWL and JWLB equations of state, as well as parameterization methodology. Two methods of parameter calibration have been used to date: empirical calibration to cylinder test data and formal optimization using JAGUAR thermo-chemical predictions. An analytic cylinder test model that uses JWL or JWLB equations of state has been developed, which provides excellent agreement with high rate continuum modeling. This analytic cylinder model is used either as part of the formal optimization or for post parameterization comparison to cylinder test data.
\end{abstract}

Keywords: blast, explosives, equation of state, modelling.

\section{Introduction}

Structure geometric configuration and response can be strongly coupled to blast loading particularly for close-in blast loading configurations. As a result, high 
rate continuum modeling is being increasingly applied to directly resolve both the blast profiles and structural response. Modeling the structural response to blast relies on accurate descriptions of the blast loading pressure profiles. When high rate continuum modeling is directly applied for the blast calculation, the explosive produced blast profile is calculated using detonation modeling of the high explosive event. In this modeling, the equation of state for the detonation products is the primary modeling description of the work output from the explosive that causes the subsequent air blast. The Jones-Wilkins-Lee (JWL) equation of state for detonation products is probably the currently most used equation of state for detonation and blast modeling. The Jones-Wilkins-LeeBaker (JWLB) equation of state is an extension of the JWL equation of state that we commonly use. The purpose of this paper is to provide a thermodynamic and mathematical background of the JWL and JWLB equations of state, as well as parameterization methodology.

\section{JWL equation of state}

The JWL thermodynamic equation of state [1] was developed to provide an accurate description of high explosive products expansion work output and detonation Chapman-Jouguet state. For blast applications, it is vital that the total work output from the detonation state to high expansion of the detonation products be accurate for the production of appropriate blast energy. The JWL mathematical form is:

$$
P=\mathrm{A}\left(1-\frac{\omega}{\mathrm{R}_{1} V^{*}}\right) e^{-\mathrm{R}_{1} V^{*}}+\mathrm{B}\left(1-\frac{\omega}{\mathrm{R}_{2} V *}\right) e^{-\mathrm{R}_{2} V *}+\frac{\omega E}{V *}
$$

where $V^{*}$ is the relative volume, $E$ is the product of the initial density and specific internal energy and $\omega$ is the Gruneisen parameter. The equation of state is based upon a first order expansion in energy of the principle isentrope. The JWL principle isentrope form is:

$$
P s \equiv \mathrm{A} e^{-\mathrm{R}_{1} V^{*}}+\mathrm{B} e^{-\mathrm{R}_{2} V^{*}}+\mathrm{C} V^{*}-(\omega+1)
$$

For JWL, the Gruneisen parameter is defined to be a constant:

$$
\left.\omega \equiv \frac{V^{*} d P}{d E}\right|_{V^{*}}
$$

Energy along the principle isentrope is calculated through the isentropic identity:

$$
d E_{s}=-P_{s} d V^{*} \Rightarrow E_{s}=\frac{A}{R_{1}} e^{-R_{1} V^{*}}+\frac{B}{R_{2}} e^{-R_{2} V^{*}}+\frac{C}{\omega V^{*} \omega}
$$


This relationship defines the internal energy referencing for consistency, so that the initial internal energy release is:

$$
\Rightarrow E_{0}=E_{C J}-\frac{1}{2} P_{C J}\left(V_{0}^{*}-V_{C J}^{*}\right)
$$

The general equation of state is derived from the first order expansion in energy of the principle isentrope:

$$
P=P_{S}+\left.\frac{d P}{d E}\right|_{V^{*}}\left(E-E_{S}\right)=P_{S}+\frac{\omega}{V^{*}}\left(E-E_{S}\right)
$$

(2), (4), (6)

$$
\Rightarrow P=\mathrm{A}\left(1-\frac{\omega}{\mathrm{R}_{1} V *}\right) e^{-\mathrm{R}_{1} V *}+\mathrm{B}\left(1-\frac{\omega}{\mathrm{R}_{2} V *}\right) e^{-\mathrm{R}_{2} V *}+\frac{\omega E}{V *}
$$

From eqns (4) and (5) it can be seen the E0 represents the total work output along the principle isentrope. For blast, this would represent the total available blast energy from the explosive.

\section{JWLB equation of state}

The JWLB thermodynamic equation of state [2] is an extension of the JWL equation of state. JWLB was developed to more accurately describe overdriven detonation, while maintaining an accurate description of high explosive products expansion work output and detonation Chapman-Jouguet state. The equation of state is more mathematically complex than the Jones-Wilkins-Lee equation of state, as it includes an increased number of parameters to describe the principle isentrope, as well as a Gruneisen parameter formulation that is a function of specific volume. The increased mathematical complexity of the JWLB high explosive equations of state provides increased accuracy for practical problems of interest. The JWLB mathematical form is:

$$
\begin{gathered}
P=\sum_{n} A_{i}\left(1-\frac{\omega}{R_{i} V^{*}}\right) e^{-R_{i} V^{*}}+\frac{\lambda E}{V^{*}} \\
\lambda=\sum_{i}\left(A \lambda i V^{*}+B \lambda i\right) e^{-R \lambda i V^{*}}+\omega
\end{gathered}
$$

where $V^{*}$ is the relative volume, $E$ is the product of the initial density and specific internal energy and $\lambda$ is the Gruneisen parameter. The JWL equation of state may be viewed as a subset of the JWLB equation of state where two inverse exponentials are used to describe the principle isentrope $(\mathrm{n}=2)$ and the Gruneisen parameter is taken to be a constant $(\lambda=\omega)$. 


\section{Analytic cylinder model}

An analytic cylinder test model that uses JWL or JWLB equations of state has been developed, which provides excellent agreement with high rate continuum modeling. Gurney formulation has often been used for high explosive material acceleration modeling [3], particularly for liner acceleration applications. The work of Taylor [4] provides a more fundamental methodology for modeling exploding cylinders, including axial flow effects by Reynolds hydraulic formulation. A modification of this method includes radial detonation product flow effects and cylinder thinning. The modifications were found to give better agreement with cylinder expansion finite element modeling [5]. One method of including radial flow effects is to assume spherical surfaces of constant thermodynamic properties and mass flow in the detonation products. The detonation products mass flow is assumed to be in a perpendicular direction to the spherical surfaces. A diagram of a products constant spherical surfaces cylinder expansion due to high explosive detonation is presented in Figure 1.

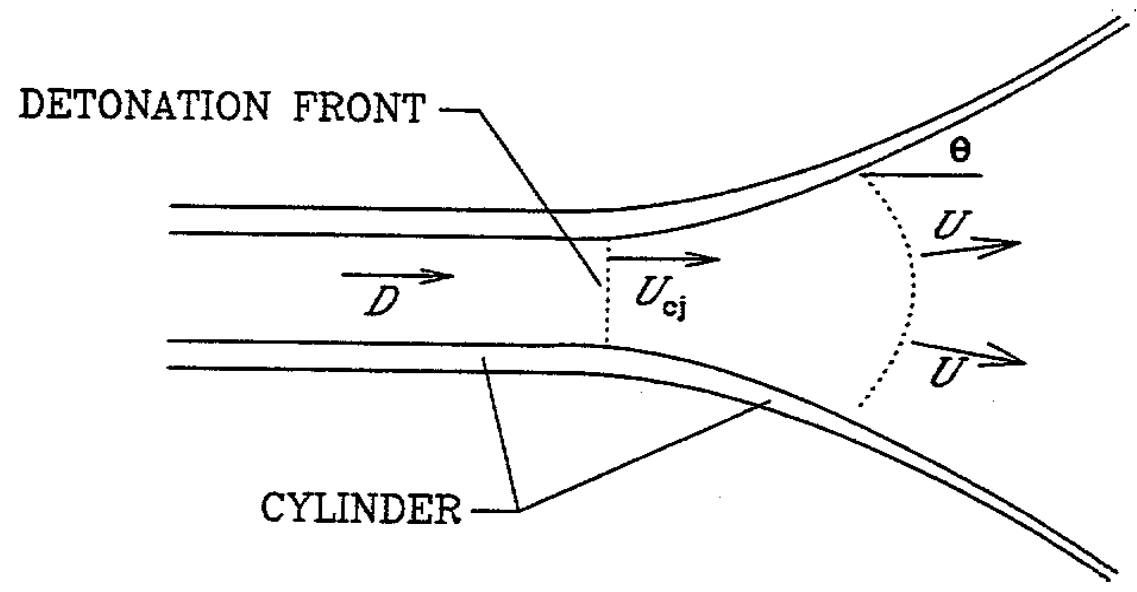

Figure 1: Analytic cylinder test model.

It should be noted that flow velocities are relative to the detonation velocity, D. If constant detonation product properties are assumed across spherical surfaces, the following model results using the JWLB thermodynamic equation of state

Mass:

Axial Momentum:

$$
\rho_{\mathrm{cj}} U_{c j} \mathrm{~A}_{0}=\rho U A
$$

$$
P_{c j} r_{0}^{2}-P r^{2}=\frac{m}{\pi} D^{2} \cos \Theta-\frac{m}{\pi} D^{2}+\rho U^{2} r^{2}-\rho_{c j} U_{c j}^{2} r_{0}^{2}
$$


Energy:

$$
\rho_{\mathrm{cj}} U_{c j} \mathrm{~A}_{0}\left(\frac{\mathrm{U}_{\mathrm{cj}}^{2}}{2}+e_{\mathrm{cj}}\right)+P_{c j} U_{c j} A_{0}=\rho U A\left(\frac{U^{2}}{2}+e\right)+P U A
$$

Principle Isentrope:

$$
P=\sum_{i} A_{i} e^{\frac{-R_{i} \rho_{0}}{\rho}}+C\left(\frac{\rho_{0}}{\rho}\right)^{-(\omega+1)}, \quad \mathrm{de}=-\operatorname{Pd}\left(\frac{1}{\rho}\right)
$$

Taylor Angle:

$$
v=2 D \sin \frac{\Theta}{2}
$$

Spherical Area:

$$
A=\pi r^{2} \frac{2(1-\cos \Theta)}{\sin ^{2} \Theta}
$$

The final equation set used for solution is:

$$
\begin{gathered}
(4) \Rightarrow P=\sum_{i} A_{i} e^{\frac{-R_{i} \rho_{0}}{\rho}}+C\left(\frac{\rho_{0}}{\rho}\right)^{-(\omega+1)} \\
(4) \Rightarrow e_{c j}-e=\sum_{i} \frac{A_{i}}{\rho_{0} \mathrm{R}_{\mathrm{i}}}\left(e^{\frac{-R_{i} \rho_{0}}{\rho_{c j}}}-e^{\left.\frac{-R_{i} \rho_{0}}{\rho}\right)}\right. \\
+\frac{C}{\omega \rho_{0}}\left[\left(\frac{\rho_{0}}{\rho_{c j}}\right)^{-\omega}-\left(\frac{\rho_{0}}{\rho}\right)^{-\omega}\right] \\
(3) \Rightarrow \frac{U^{2}}{2}=\frac{U_{c j}^{2}}{2}+\frac{P_{c j}}{\rho_{c j}}-\frac{P}{\rho}+e_{c j}-e \\
(2) \Rightarrow \frac{v^{2}}{2}=\left[P\left(\frac{r}{r_{0}}\right)^{2}-P_{c j}+\rho\left(\frac{r}{r_{0}}\right)^{2} U^{2}-\rho_{c j} U_{c j}^{2}\right] \frac{C}{m \rho_{0}} \\
\text { (1), (5), (6) } \Rightarrow \rho=\frac{\rho_{c j} U_{c j}}{U\left(\frac{r}{r_{0}}\right)^{2}}\left[1-\left(\frac{v}{2 D}\right)^{2}\right]
\end{gathered}
$$

This set of equations is solved for a given area expansion, $\left(\mathrm{r} / \mathrm{r}_{0}\right)^{2}$ using Brent's method [6]. The spherical surface approach has been shown to be more accurate for smaller charge to mass ratios without any loss of agreement at larger charge 
to mass ratios. It should be recognized that this analytic modeling approach neglects initial acceleration due to shock processes [7] and is therefore anticipated to be more accurate as the initial shock process damps out. The model as expressed does not consider the fact that the cylinders thin during radial expansion. One simple way to account for this wall thinning is to assume that the wall cross sectional area remains constant and $\mathrm{r}$ and $\mathrm{v}$ represents the inside radius and inside surface wall velocity.

$$
v_{\text {out }}=v \frac{r_{\text {in }}}{r_{\text {out }}} ; r_{\text {out }}^{2}=r_{\text {in }}^{2}+r_{\text {out }}^{2}-r_{\text {in }}^{2}
$$

\section{Eigenvalue analytic cylinder model}

High explosives are often aluminized for blast enhancement. Eigenvalue detonations are observed for some aluminized explosives [9]. For this reason, it was of interest to develop a modified analytic cylinder test model that provides a description of the detonation products isentropic expansion from the eigenvalue detonation weak point, rather than from the Chapman-Jouguet state. It was found that the most straight forward method of implementation of an eigenvalue detonation analytic cylinder model was to refit the isentrope associated with the eigenvalue weak point using eqn (13). In this way, equations 1-11 remain correct, except that eigenvalue weak point is used, rather than the ChapmanJouguet state. With this approach, it is important to realize that the weak-point isentrope fit is not the same as the principle isentrope fit. The final form is:

$$
\begin{gathered}
P=\sum_{i} A_{w i} e^{\frac{-R_{w i} \rho_{0}}{\rho}}+C_{w}\left(\frac{\rho_{0}}{\rho}\right)^{-(\omega+1)} \\
e_{w}-e=\sum_{i} \frac{A_{w i}}{\rho_{0} \mathrm{R}_{\mathrm{wi}}}\left(e^{\frac{-R_{w i} \rho_{0}}{\rho_{w}}}-e^{\left.\frac{-R_{w i} \rho_{0}}{\rho}\right)}\right. \\
+\frac{C_{w}}{\omega \rho_{0}}\left[\left(\frac{\rho_{0}}{\rho_{w}}\right)^{-\omega}-\left(\frac{\rho_{0}}{\rho}\right)^{-\omega}\right] \\
\frac{U^{2}}{2}=\frac{U_{w}^{2}}{2}+\frac{P_{w}}{\rho_{w}}-\frac{P}{\rho}+e_{w}-e \\
\frac{P\left(\frac{r}{r_{0}}\right)^{2}-P_{w}}{2}=\left[\begin{array}{c}
C \\
+\rho\left(\frac{r}{r_{0}}\right)^{2} U^{2}-\rho_{w} U_{w}^{2}
\end{array}\right] \frac{C}{m \rho_{0}}
\end{gathered}
$$




$$
\rho=\frac{\rho_{w} U_{w}}{U\left(\frac{r}{r_{0}}\right)^{2}}\left[1-\left(\frac{v}{2 D_{w}}\right)^{2}\right]
$$

\section{High rate continuum modeling comparison}

ALE3D high rate continuum modeling, Figure 2, was compared to analytic cylinder test modeling using identical JWLB equations of state for TNT, LX-14 and PAX-30 for 1 inch diameter charges and 0.1 inch and 0.2 inch thick copper cylinders.

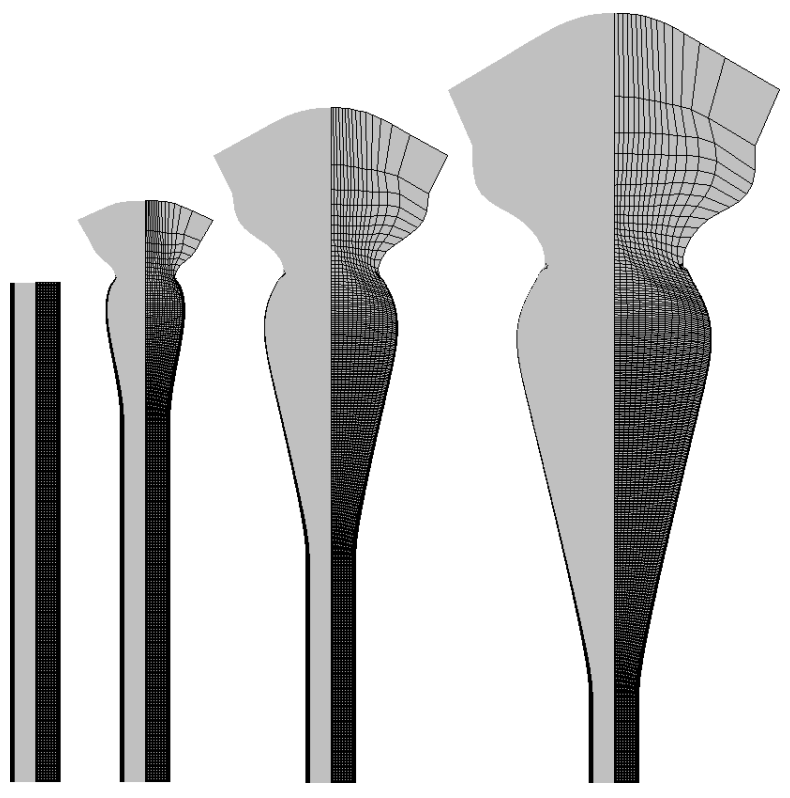

Figure 2: $\quad$ Modeling at $10 \mu$ intervals for 0.1 " thick copper cylinder.

Figures 3,4 and 5 present the comparison of the analytic cylinder test model to the ALE3D modeling for TNT, LX-14 and PAX-30 respectively. The analytic cylinder model slightly under predicts the velocities at 2 and 3 inside area expansions, but is in very close agreement by 6 and 7 inside area expansions. This is consistent with the fact that this analytic modeling approach neglects initial acceleration due to shock processes. Strong shock effects are typically observed in the 2 to 3 volume expansion region and are practically damped out by 6 volume expansions, where very close agreement between the analytic model and ALE3D results are observed. 
154 Structures Under Shock and Impact XI

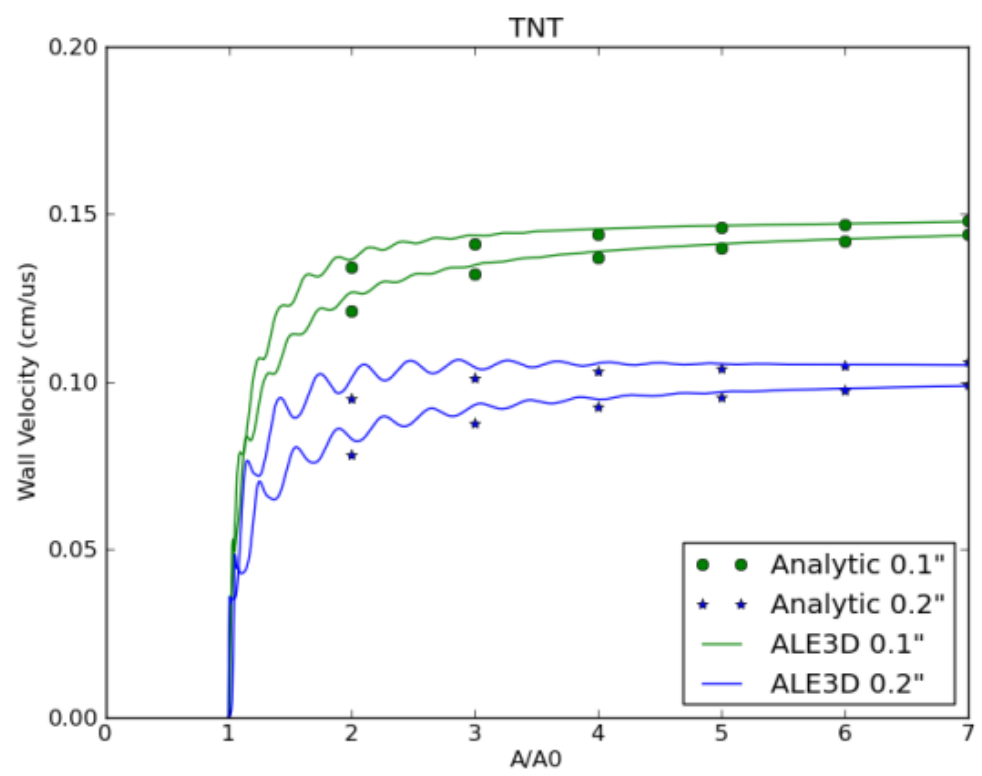

Figure 3: $\quad$ TNT cylinder analytic model versus ALE3D.

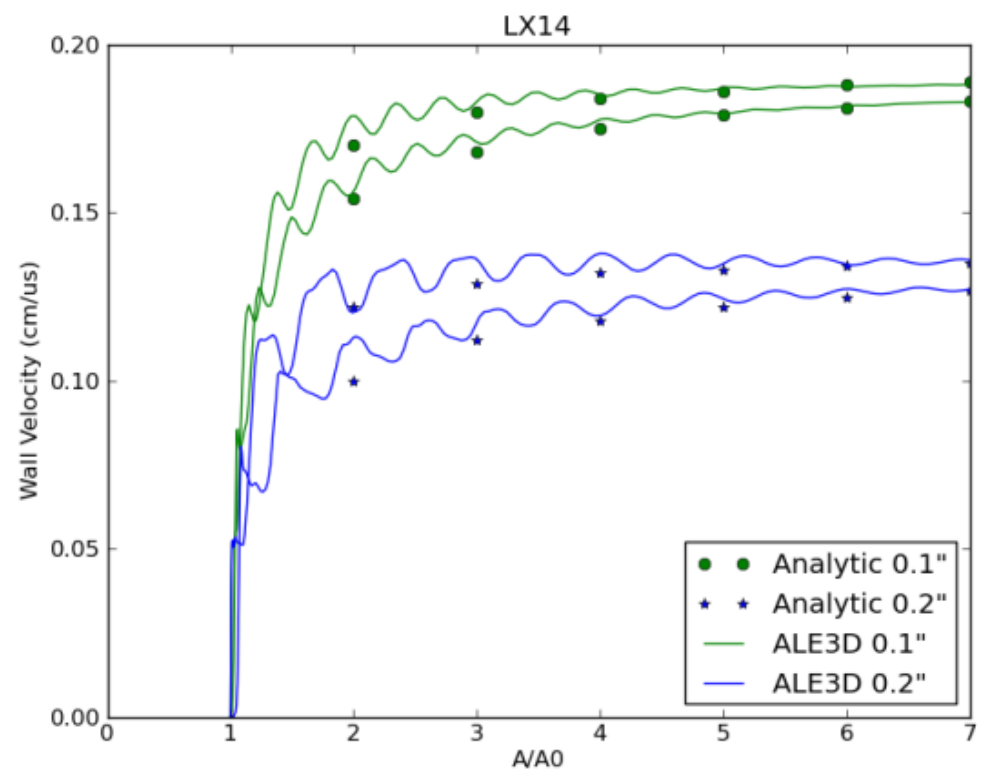

Figure 4: $\quad$ LX-14 cylinder analytic model versus ALE3D. 


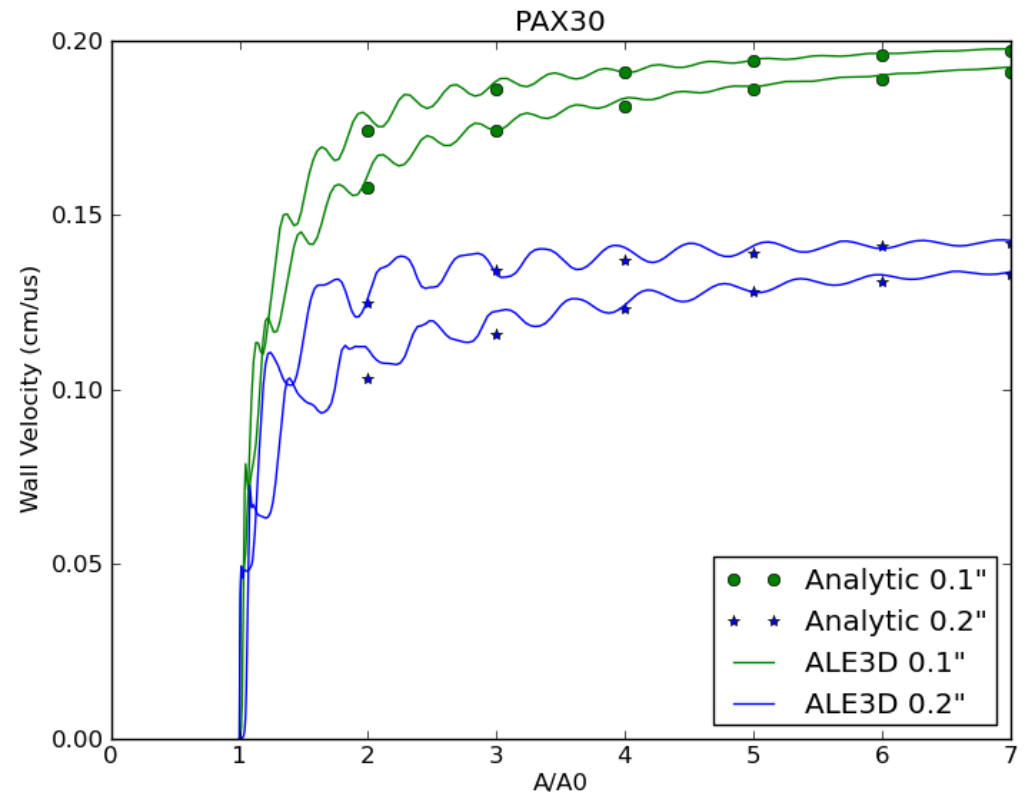

Figure 5: $\quad$ PAX-30 cylinder analytic model versus ALE3D.

\section{Parameterization}

We use two methods of parameterization are used to calibrate the JWL and JWLB constants. Both employ non-linear variable metric optimization techniques [2] for the parameterization process. In the first method [2], the equation of state parameters are optimized to reproduce the experimental cylinder velocities using the analytic cylinder test model, as well as to reproduce a desired Chapman-Jouguet detonation velocity and pressure. Typically, the total principle isentrope work output $\mathrm{E}_{0}$ is also fixed to provide a desired total blast output. The cylinder velocities are used in a cost function to be minimized, whereas the Chapman-Jouguet state and $\mathrm{E}_{0}$ are treated as equality constraints. The second method of parameterization [8] is to directly fit the predicted pressure and Gruneisen parameter versus specific volume behavior predicted by the thermo-chemical equation of state computer program JAGUAR. Formal non-linear optimization is used for the parameterization procedure. The LX-14 high energy explosive example presented in Figure 4 used the technique of parameterization for the JWLB equation of state. JWL and JWLB equation of states were parameterized for LX-14 using the JAGUAR predictions and nonlinear optimization routines. The resulting JWL and JWLB equations of state were then used to model a standard 1.2 inch outside diameter and 1 inch inside diameter copper cylinder test ( 0.1 " thick wall) and compared to experimental data using the analytic cylinder test model. Table 1 presents the resulting outside cylinder velocity results at different inside cylinder cross sectional areas. The results clearly show the improved agreement to experimental data obtained when 
using the more mathematically complex JWLB mathematical form. The improved agreement is attributed to the improved agreement to the JAGUAR predicted detonation products behavior that is achieved using the JWLB form.

Table 1: LX-14 JWL and JWLB cylinder test velocity predictions $(\mathrm{Km} / \mathrm{s})$ compared to experimental data.

\begin{tabular}{cccc}
\hline & & \multicolumn{2}{c}{ ANALYTIC CYLINDER } \\
A/AO & EXPERIMENTAL & JWL & JWLB \\
2 & 1.505 & 1.562 & 1.519 \\
3 & 1.664 & 1.705 & 1.667 \\
4 & 1.745 & 1.759 & 1.738 \\
5 & 1.791 & 1.79 & 1.78 \\
6 & 1.817 & 1.812 & 1.807 \\
7 & 1.833 & 1.828 & 1.826 \\
& & & \\
2 & & 3.787 & 0.930 \\
3 & & 2.464 & 0.180 \\
4 & & 0.802 & 0.401 \\
5 & & 0.056 & 0.614 \\
6 & & 0.275 & 0.550 \\
7 & & 0.273 & 0.382 \\
AVERAGED ERROR (\%) & $\mathbf{1 . 2 7 6}$ & $\mathbf{0 . 5 1 0}$ \\
\hline
\end{tabular}

Table 2: $\quad$ PAX-30 JWL cylinder test predictions compared to experiments.

\begin{tabular}{ccccc}
\hline A/A0 & EXPERIMENTAL & JWL & JWLB & JWLB w-point \\
2 & 1.499 & 1.599 & 1.55 & 1.541 \\
3 & 1.682 & 1.759 & 1.702 & 1.703 \\
4 & 1.774 & 1.823 & 1.780 & 1.779 \\
5 & 1.827 & 1.862 & 1.831 & 1.825 \\
6 & 1.859 & 1.89 & 1.868 & 1.856 \\
7 & 1.883 & 1.911 & 1.897 & 1.879 \\
& & & & \\
& & & $\%$ ERROR & \\
2 & & 6.6711 & 3.4023 & 2.8019 \\
3 & & 4.5779 & 1.1891 & 1.2485 \\
4 & & 2.7621 & 0.3157 & 0.2818 \\
5 & & 1.9157 & 0.2189 & 0.1095 \\
6 & & 1.6676 & 0.4841 & 0.1614 \\
7 & & 1.4870 & 0.7435 & 0.2124 \\
AVERAGED ERROR (\%) & $\mathbf{3 . 1 8 0 2}$ & $\mathbf{1 . 0 5 8 9}$ & $\mathbf{0 . 8 0 2 6}$ \\
\hline
\end{tabular}


Similar to the LX-14, JWL and JWLB equation of states were also parameterized for PAX-30 using the JAGUAR predictions and non-linear optimization routines. The resulting JWL and JWLB equations of state were again used to model a standard 1.2 inch outside diameter and 1 inch inside diameter copper cylinder test $(0.1$ " thick wall) and compared to experimental data using the analytic cylinder test model. However, PAX-30 is an aluminized explosive that is known to produce eigenvalue detonations [9]. Table 2 presents the resulting outside cylinder velocity results at different inside cylinder cross sectional areas. Again, the results clearly show the improved agreement to experimental data obtained when using the more mathematically complex JWLB mathematical form. The results also show a slight improvement by using the eigenvalue analytic cylinder model that represents expansion from the weak point (w-point). Table 3 presents JWLB equation of state parameters for TNT, LX-14 and PAX-30, which were used in this study.

Table 3: JWLB equation of state parameters for TNT, LX-14 and PAX-30.

\begin{tabular}{|c|c|c|c|c|c|}
\hline & TNT & \multicolumn{2}{|c|}{ PAX-30 } & \multicolumn{2}{c|}{ LX-14 } \\
\hline$\rho$ (g/cc) & 1.6300 & 1.885 & 1.909 & 1.820 & 1.8350 \\
\hline E0 (Mbar) & 0.0657 & 0.13568 & 0.1376 & 0.102195 & 0.1032 \\
\hline $\mathrm{D}(\mathrm{cm} / \mu \mathrm{s})$ & 0.6817 & $0.8342^{*}$ & $0.8429^{*}$ & 0.86337 & 0.8691 \\
\hline $\mathrm{P}(\mathrm{Mbar})$ & 0.1930 & $0.2419^{*}$ & $0.2464^{*}$ & 0.33529 & 0.3418 \\
\hline $\mathrm{A} 1$ (Mbar) & 399.2140 & 406.224 & 405.3810 & 399.995 & 399.1910 \\
\hline $\mathrm{A} 2$ (Mbar) & 56.2911 & 135.309 & 14.8887 & 20.1909 & 52.1951 \\
\hline $\mathrm{A} 3$ (Mbar) & 0.8986 & 1.5312 & 1.49138 & 1.42441 & 1.59892 \\
\hline $\mathrm{A} 4$ (Mbar) & 0.0092 & 0.006772 & 0.0076 & 0.02273 & 0.0249 \\
\hline $\mathrm{R} 1$ & 28.0876 & 26.9788 & 13.2982 & 13.93720 & 27.4041 \\
\hline $\mathrm{R} 2$ & 9.7325 & 10.6592 & 8.0204 & 7.230140 & 8.4331 \\
\hline $\mathrm{R} 3$ & 2.5309 & 2.52342 & 2.4942 & 2.558910 & 2.6293 \\
\hline $\mathrm{R} 4$ & 6.9817 & 0.335585 & 0.3566 & 0.736406 & 0.7498 \\
\hline $\mathrm{C}(\mathrm{Mbar})$ & 0.0076544 & 0.013561 & .0135749 & 0.011016 & 0.385366 \\
\hline$\omega$ & 0.345920 & 0.234742 & 0.234664 & 0.384733 & .0110204 \\
\hline $\mathrm{A}^{\lambda} 1$ & 58.2649 & 72.6781 & 66.6542 & 41.71970 & 68.6476 \\
\hline $\mathrm{A}^{\lambda} 2$ & 6.1981 & 5.64752 & 5.7776 & 6.83632 & 6.7497 \\
\hline $\mathrm{B}^{\lambda} 1$ & 2.9036 & 2.8728 & 3.1440 & 6.42909 & 4.1338 \\
\hline $\mathrm{B}^{\lambda} 2$ & -3.2455 & -3.10754 & -3.2552 & -4.47655 & -4.4607 \\
\hline $\mathrm{R}^{\lambda} 1$ & 25.5601 & 27.8109 & 25.5996 & 25.72540 & 26.2448 \\
\hline $\mathrm{R}^{\lambda} 2$ & 1.7034 & 1.71375 & 1.7099 & 1.71081 & 1.6977 \\
\hline
\end{tabular}

* Eigenvalue weak point detonation state (not the Chapman-Jouguet state).

\section{Conclusions}

An analytic cylinder test model has been developed by ARDEC for explosive equation of state calibration and verification. The analytic model was based on adiabatic expansion along the principle isentrope from the Chapman-Jouguet 
state. Additionally, an eigenvalue extended analytic cylinder expansion model has been developed based on isentropic expansion from the detonation eigenvalue weak point, rather than from the Chapman-Jouguet state. High explosives often include additive aluminium for blast effects. This eigenvalue model is applicable to Al based explosives, such as PAX-30, that exhibit eigenvalue detonations. The results for these explosives show only a very small reduction of explosive work output for eigenvalue detonations compared to Chapman-Jouguet detonations. This is due to the fact that the Chapman-Jouguet principle isentrope and eigenvalue weak point isentrope lie very close to each other. Excellent agreement between the analytic cylinder test and high rate continuum modeling predicted cylinder velocities is achieved when using the same JWL or JWLB parameters.

\section{References}

[1] Lee, E.L., Hornig, C. \& Kury, J.W., "Adiabatic Expansion of High Explosive Detonation Products", Lawrence Livermore Laboratory, Rept. UCRL-50422 (1968).

[2] Baker, E.L. "An Application of Variable Metric Nonlinear Optimization to the Parameterization of an Extended Thermodynamic Equation of State", Proceedings of the Tenth International Detonation Symposium, Edited by J. M. Short and D. G. Tasker, Boston, MA, pp. 394-400, July 1993.

[3] Gurney, R. W., "The Initial Velocities of Fragments from Bombs, Shells, and Grenades," BRL Report 405, U.S. Army Ballistic Research Lab, 1943.

[4] Taylor, G. I., Analysis of the Explosion of a Long Cylindrical Bomb Detonated at One End, Scientific Papers of Sir G. I. Taylor, Vol 111:2770286, Cambridge University Press (1963), 1941.

[5] Baker, E.L., "Modeling and Optimization of Shaped Charge Liner Collapse and Jet Formation", Picatinny Arsenal Technical Report ARAED-TR92017, January 1993.

[6] Brent, R., Algorithms for Minimization without Derivatives. Prentice-Hall, Englewood Cliffs, NJ, 1973. Reprinted by Dover Publications, Mineola, New York, January 2002.

[7] Backofen, J.E., "Modeling a Material's Instantaneous Velocity during Acceleration Driven by a Detonation's Gas-Push", 2005: Proceedings of the Conference of the American Physical Society Topical Group on Shock Compression of Condensed Matter, AIP Conf. Proc., July 28, 2006, Volume 845, pp. 936-939.

[8] Baker, E.L. \& L.I. Stiel, "Improved Cylinder Test Agreement with JAGUAR Optimized Extended JCZ3 Procedures", Proceedings of the International Workshop on New Models and Numerical Codes for Shock Wave Processes in Condensed Media, St. Catherines College, Oxford, UK, September 1997.

[9] Baker, E.L., Stiel, L.I., Capellos, C., Balas, W. \& Pincay, J., "Combined Effects Aluminized Explosives", Proceedings of the International Ballistics Symposium, New Orleans, LA, USA, 22-26 September 2008. 